\title{
La prévention des accidents du travail
}

\author{
LA LOI DU 6 DÉCEMBRE 1976 : \\ PRINCIPALES DISPOSITIONS, \\ INFLUENCE RÉCIPROQUE DES DIVERS RISQUES \\ ET COLLABORATION NÉCESSAIRE DES SERVICES
}

\author{
A. PACHET $(*)$, \\ ancien Directeur du travail
}

(Manuscrit reçu le 28 juin 1977)

\begin{abstract}
RÉSUMÉ
L'auteur invite les spécialistes de la radioprotection à bien connaître la réglementation de sécurité non nucléaire, et insiste sur la liaison indispensable entre tous ceux qui sont chargés des questions de sécurité en raison même de l'aggravation réciproque des risques de tous ordres.

Principales dispositions de la loi de 1976 : Extension de l'application du Code du Travail à l'agriculture, le secteur public reste exclu; nouvelles règles pour les opérations de construction: les très gros chantiers devront avoir des collèges interentreprises de sécurité et les entreprises devront créer des C.H.S. et établir des plans de sécurité; le contrôle đes substances et des matériels dangereux est généralisé et facilité par le développement du recours aux organismes agréés. Des pénalités nouvelles sont ajoutées au Code du Travail. Ces textes provoquent de vives discussions, leur portée réelle ne sera appréciable qu'à la lumière de ce qu'en feront les magistrats. D'autres dispositions sont relatives à la formation rendue obligatoire dans certains cas, au travail au rendement, parfois facteur d'insécurité, à l'Inspection du Travail (quelques procédures aménagées), à la Sécurité Sociale (légère amélioration de ses moyens). Les dispositions pénales relatives aux responsabilités sont immédiatement applicables et préoccupent certaines catégories de personnel. Toutes les autres dispositions doivent être complétées par des décrets d'application avant d'entrer en vigueur. En conclusion, des règles de grande portée à côté d'autres de peu d'intérêt ou même contestables.
\end{abstract}

$\left(^{*}\right)$ 137, avenue La Bruyère, 94400 Vitry. 


\begin{abstract}
Health physicists are urged to get well acquainted with the regulations on nonnuclear safety; close relationships must be established between all those that are encharged with safety questions on account of the reciprocal increase of all kinds of hazards. The main provisions of the Law of 1976 are stated: extension of the applications of the Code of Labour to agriculture; new regulations for building operations-large building yards must have common safety colleges and the contractors shall set up Health and safety committes and draw up safety plans; the control of dangerous substances and materials is generalized and facilitated by the development of appeal to certified organizations. New penalties are added to the Code of Labour. Brisk discussions are raised by these texts, their actual significance will be appreciated at the light of court decisions. Other provisions are relative to training, compulsory in some cases, output work sometimes generating unsafety; Labour Inspection, Social Security. Penalty clauses about responsibilities take effect directly and are of concern for some classes of staff. All the other provisions must be supplemented by enactments.
\end{abstract}

Avant d'exposer à grands traits le contenu de la loi du 6 décembre 1976 (J.O. du 7 décembre) sur la prévention des accidents du travail, m'adressant à des spécialistes de la radioprotection, je voudrais insister sur l'action réciproque des divers risques que l'on rencontre dans le monde du travail et sur la nécessité des liaisons que cette diversité impose, en plus de tant d'autres raisons.

Pour connaître le niveau global de sécurité d'une installation, il est de bonne méthode de considérer l'ensemble des risques de celle-ci, quelle que soit leur nature. Mais la grande difficulté, pour ceux qui sont " chargés » de la sécurité des travailleurs est de bien connaître ces risques, leur nature, leur ampleur, leur action réciproque possible et les moyens d'y faire face.

Or, les produits et les procédés industriels se renouvellent et varient relativement vite et il en va de même des techniques de protection.

Il me semble important, ici, de situer le risque d'irradiation parmi les autres, et de souligner que certains d'entre eux, isolément peu graves, peuvent être un facteur d'élévation considérable du risque nucléaire. Il y eut un temps, au début du siècle, où l'utilisation industrielle de l'électricité avait fait du risque électrique l'un des plus préoccupants; il a été maîtrisé et il est rangé aujourd'hui parmi ceux qu'il est d'usage de désigner par le terme de risques classiques, par opposition au risque d'irradiation.

Il ne semble pas excessif de dire que, dès aujourd'hui, le nouveau risque nucléaire est, lui aussi, bien connu et contrôlé. Dès la fin de la seconde guerre mondiale, il a fallu s'assurer des services de l'atome sans en subir les dommages. Des raisons psychologiques et sociales se sont ajoutées aux nécessités techniques et ont conduit à un effort considérable et au développement très rapide d'une technologie particulière de la protection des travailleurs contre l'irradiation ou la contamination. Des services particuliers de protection se sont développés et ont œuvré avec succès et une réglementation publique a pris place en 1966 et 1967. 
Mais il existe d'autres branches de la sécurité et d'autres spécialistes.

Se consacrer à la sécurité du travail demande bien des qualités dont je citerai quelques-unes :

- une formation de base assez polyvalente, car la sécurité fait appel aux notions les plus diverses, physique, chimie, mécanique ... voisinant avec biologie, médecine ou psychosociologie;

- une grande ouverture d'esprit pour sortir des frontières de sa discipline initiale;

- beaucoup de désintéressement et une certaine humilité pour mettre ses connaissances à jour;

- des facultés humaines et sociales pour se faire entendre de la hiérarchie autant que du personnel, et savoir dialoguer avec ses collègues.

C'est cette dernière qualité que je voudrais souligner aujourd'hui. Le moment me paraît largement venu, pour tous ceux qui sont chargés de la sécurité des travailleurs et des installations, de se concerter de façon systématique. Quelques accidents électriques ou chimiques, par exemple, ou quelques débuts d'incendie démontrent la nécessité impérieuse de cette liaison. L'établissement des dossiers des installations nucléaires de base conduit à la même démarche. Elle vaut, d'ailleurs, pour les installations de toutes natures, quels qu'en soient l'importance, le volume ou les autres caractères, même si le risque global semble, a priori, peu grave. Il est tout aussi important, à mon avis, pour les ingénieurs et cadres de sécurité d'avoir des notions sur le risque d'irradiation, que pour les spécialistes de la radioprotection de s'initier aux règles essentielles de la sécurité non nucléaire.

C'est dans cet esprit qu'il a semblé, à certains amis de la S.F.R.P. et à moimême, que les lecteurs de la revue apprécieraient quelques informations débordant le cadre habituel. La parution de la loi de 1976 nous en donne l'occasion. Certaines de ses dispositions orienteront bien des habitudes ou des procédures du Code du Travail.

\section{LA LOI DU 6 DÉCEMBRE 1976}

Cette loi résulte d'un mouvement d'opinion que le lecteur connaît bien, d'une attitude plus stricte de certains tribunaux ainsi que des dossiers qui étaient en attente au Ministère du Travail. Mais les choix ont provoqué de très vifs débats et les textes ont été soumis à l'Assemblée et au Sénat à trois reprises entre le 13 mai et leur publication le 7 décembre 1976.

La loi nouvelle contient beaucoup de dispositions très positives, d'autres dont l'intérêt n'est pas toujours évident et quelques autres qui inquiètent un peu les cadres. A l'exception des textes sur les responsabilités et les sanctions, tous les autres doivent être complétés par des décrets et ne seront applicables qu'à la parution de ceux-ci. Cependant, les orientations sont déjà dessinées et cet exposé se propose d'en souligner les plus importantes.

vOL. $12-\mathrm{N}^{\circ} 4$ 
Les sujets sont présentés dans un ordre arbitraire et sont situés dans l'évolution de la réglementation.

\section{LE CHAMP D'APPLiCATION DU CODE dU TRAVAIL}

Le Code du Travail fut créé au début du siècle. Les dispositions d'hygiène et de sécurité ont d'abord été appliquées aux établissements industriels et commerciaux. En 1976, la loi les étend aux établissements agricoles, des décrets prévoiront leur adaptation à l'agriculture. On peut regretter, cependant, que le législateur ne soit pas allé jusqu'à étendre la protection des règles d'hygiène et de sécurité à tous les travailleurs du pays. En effet, les employés des administrations publiques, des établissements publics, et des collectivités locales n'en bénéficient pas de droit.

\section{Construction}

Un chapitre nouveau, très important, apparaît et crée deux ordres de dispositions :

$1^{\circ}$ La sécurité intégrée devient une démarche réglementaire. Cette notion est déjà bien appliquée par certains constructeurs comme ceux des grandes installations nucléaires; elle consiste à prévoir les mesures de sécurité dès la conception d'un ensemble quelconque en vue de son utilisation et de son entretien dans de bonnes conditions.

$2^{\circ}$ Les gros chantiers de construction devront désormais appliquer quelques règles dont on espère l'allègement du lourd tribut que paient les hommes du bâtiment, profession qui détient le record dans les statistiques d'accidents :

- des aménagements d'hygiène et de sécurité devront être prévus avant l'ouverture des gros chantiers;

- chaque entreprise devra élaborer un plan d'action de prévention;

- les entreprises du chantier devront assurer la coordination de leur travail de sécurité en créant un collège de sécurité.

Il faut souligner que ce chapitre concerne non seulement les entreprises du Bâtiment, mais aussi le maître d'œuvre et le maître de l'ouvrage.

\section{MATÉRIELS ET PRODUITS DANGEREUX}

Avant d'indiquer les nouvelles règles qui seront imposées aux matériels et aux produits dangereux, il est intéressant d'évoquer l'évolution des règlements.

Au début du siècle, l'importance de quelques risques a conduit le législateur à prendre des dispositions pour y faire face. Il y eut ainsi une série de décrets en 1913. A cette époque, seule l'entreprise utilisatrice des appareils ou des produits se voyait imposer des règles de protection des travailleurs.

Après la seconde guerre mondiale, on va vouloir empêcher la mise sur le marché de matériels dangereux, et l'on va procéder par homologation des 
matériels à vendre. L'autorisation accordée pour chaque machine permet un contrôle très efficace mais la procédure est bien lourde. Puis, on s'attaque aux produits dangereux et, en 1950 et 1951, l'étiquetage de quelques substances très dangereuses est imposé aux vendeurs. Cette procédure se révèle très efficace; en 1972, elle est élargie à plus de 500 produits et s'impose à la fois aux vendeurs et aux utilisateurs.

Une dernière étape est franchie en 1973, puis avec la loi de 1976 : le champ de contrôle des matériels et des produits dangereux est étendu à toutes les étapes depuis la fabrication ou l'importation et la vente, jusqu'à l'utilisation et même l'élimination. La procédure d'homologation sera maintenue pour certaines machines, mais complétée pour les autres par un système de normalisation défini avec le concours des organismes professionnels. Les organismes agréés voient, d'autre part, leur action largement étendue. Ils ont été créés pour opérer après mise en demeure de l'Inspecteur du Travail. Les industriels ont pris l'habitude de recourir par abonnement aux services de ces organismes particulièrement bien outillés et compétents. La loi de 1976 élargit ce système de contrôle technique qui vient épauler l'action du contrôle administratif.

Dégageons maintenant les grandes lignes des règles établies pour les matériels dangereux d'abord puis pour les produits dangereux.

\section{MATÉRIELS DANGEREUX}

La procédure d'homologation a permis un contrôle très efficace de quelques machines les plus dangereuses comme les machines à bois, ou les presses automatiques. Les nouvelles dispositions conduiront à élargir la surveillance à tous les matériels dangereux et en toutes circonstances. Trois types de dispositions sont prévus :

$1^{\circ}$ pour les matériels les plus dangereux : les décrets prévoiront les conditions d'hygiène et de sécurité et la procédure de vérification;

$2^{\circ}$ pour les autres matériels, des mesures générales seront définies et l'on aura vraisemblablement recours à un système de normes établies avec le concours des organismes professionnels patronaux et ouvriers;

$3^{\circ}$ une procédure d'urgence est enfin définie, elle complètera les moyens déjà donnés au Comité d'Hygiène et de Sécurité (C.H.S.) et à l'Inspecteur du Travail en cas de danger jugé grave et imminent.

Le législateur a été sage, ici, d'exclure l'idée d'une action directe - ordre d'arrêt d'une installation par exemple - du représentant du personnel ou de l'Inspecteur du Travail qui doit saisir le juge des référés.

\section{SUBSTANCES ET PRÉPARATIONS DANGEREUSES}

Comme pour les machines, le contrôle des produits dangereux étend le champ de surveillance et améliore les procédures.

1. Les nouvelles règles imposent de contrôler toutes les substances ou préparations dangereuses pour les travailleurs et à toutes les étapes, depuis l'importation ou la fabrication jusqu'à la vente ou l'usage. 
2. Les procédures :

a) Avant la vente, le recours à l'avis d'organismes agréés, préalable à la mise en vente, sera généralisé.

b) Après la vente, il sera désormais possible :

- de faire analyser des produits par des organismes agréés;

- d'interdire leur vente;

- d'interdire leur emploi.

Remarquons, ici, que la France ne s'engage pas encore dans le contrôle de l'atmosphère des locaux de travail par la définition de limites admissibles, comme cela se pratique dans de nombreux pays étrangers. Les techniques actuelles d'analyse permettent cette surveillance; j'estime que cette omission est très regrettable; on en est réduit à utiliser les normes publiées, à titre indicatif, par I'I.N.R.S. (Institut National de Recherche sur la Sécurité).

\section{ORGANISMES PROFESSIONNELS}

Des pouvoirs assez importants pourront être donnés à certains organismes professionnels en matière de formation, d'études, de proposition de réglementation. Espérons que cette voie ne conduise pas à ce que l'intérêt de certaines professions aille à l'encontre de l'intérêt général.

\section{COMITÉS D’hygIÈnE ET DE SÉCURITÉ (C.H.S.)}

Certains parlementaires, sans remettre en cause l'action positive de l'O.P.P.B.T.P., organisme des professions du Bâtiment chargé en particulier de jouer le rôle des C.H.S. au plan national, ont obtenu que l'on prévoit la création de C.H.S. dans certains chantiers du Bâtiment. Cette disposition devra s'articuler avec la création des Collèges de Sécurité. Le même élargissement des C.H.S. est décidé pour les Mines.

Il reste que les règles relatives au C.H.S. sont insuffisantes et désordonnées. Créée en 1947, l'institution n'est pas encore un succès. Et le fait de confier aux organismes professionnels, que je viens de citer, le soin d'assurer le rôle des C.H.S. pour les petites entreprises, s'il peut être positif, ne réglera pas toutes les difficultés. Les dialogues et la concertation, tant vantés, doivent être encouragés. C'est sur place que les questions de sécurité apparaissent et doivent se régler. J'aurais donc préféré un élargissement des moyens d'expression des travailleurs plutôt qu'un recours à des organes extérieurs. Il faut pour cela améliorer le fonctionnement du C.H.S. et donner aux représentants du personnel les moyens de s'exprimer grâce à :

- une formation particulière,

- une protection identique à celle des délégués du personnel, actuellement réservée aux C.H.S. des seules entreprises de plus de 300 salariés.

Rappelons que les délégués du personnel sont chargés des questions de sécurité, entre autres, et qu'il ne serait pas malsain de leur assurer une formation de sécurité. Tout le monde y gagnerait. 


\section{FORMATION}

Une obligation de formation de sécurité est ouverte, à la charge des entreprises et indépendamment des droits à l'éducation permanente : 21 jours;

$1^{\text {o }}$ à l'embauche, en cas de modification de poste et après une absence de

$2^{\circ}$ pour certains établissements à hauts risques.

\section{TravaIL AU RENDEMENT}

La loi de 1976 tend à limiter progressivement les risques qui résultent des cadences, des rythmes de travail ou du travail par équipes. Dans le même esprit, en cas de modification de poste, lorsque le travail est payé au rendement, le salaire moyen antérieur devra être maintenu pendant 2 semaines.

\section{L'INSPECTION DU TRAVAIL}

Les pouvoirs de l'Inspection du Travail ont été évoqués avec beaucoup d'insistance devant les deux chambres du Parlement. Les orateurs de tous horizons en ont souligné l'importance, l'utilité et le manque de moyens. Il n'en résulte cependant que quelques dispositions de peu de portée aménageant la procédure de mise en demeure ou de recours.

L'amélioration des conditions du travail devrait, à mon avis, résulter de quelques démarches complémentaires :

$1^{\circ}$ Développer les moyens de prévention à l'intérieur de l'entreprise :

- appliquer plus strictement les règles définies pour les Comités d'entreprises, les délégués et les C.H.S.; les Inspecteurs du Travail seraient alors allégés de bien des contestations;

- développer la formation de sécurité des divers représentants du personnel;

- créer un statut des ingénieurs et des cadres de sécurité.

L'ensemble de ces mesures internes à l'entreprise constituerait un complément très utile aux actions extérieures, qu'il s'agisse des contrôles administratifs ou des aides techniques.

\section{$2^{\circ}$ Accroître les moyens d'action des Inspecteurs du Travail :}

Les possibilités de contrôle seraient largement accrues par l'augmentation du nombre d'inspecteurs, c'est évident, mais aussi il serait bien d'accroître hardiment le nombre des contrôleurs du travail et le personnel administratif des services. Les moyens matériels, enfin, sont bien trop parcimonieux.

\section{$3^{\circ}$ Généraliser effectivement l'Inspection du Travail :}

L'idée a été avancée, une fois de plus, de former des inspecteurs spécialisés pour faire face au développement de techniques particulières. Le Code du Travail a déjà choisi le système de l'inspecteur, généraliste du Droit du Travail, 
assisté d'aides techniques : ingénieurs, médecins, organismes agréés. C'est cette voie que la loi de 1976 confirme heureusement en développant le recours aux organismes agréés. Il restera à doter le Ministère du Travail des crédits nécessaires pour assurer ces missions.

Enfin, il y a encore trop de corps de fonctionnaires chargés des fonctions d'Inspecteur du Travail. Dans certains cas, le contrôle est plus théorique que réel, faute de formation ou de moyens ou parce que cette conception est mauvaise.

La protection de tous les travailleurs de ce pays devrait être assurée de façon égale, ce qui implique, non seulement l'application du Code du Travail à tous, mais un contrôle efficace et coordonné des entreprises ou organismes de toutes natures, publics ou privés.

\section{SÉCURITÉ SOCIALE}

Quelques dispositions améliorent les possibilités de la Sécurité Sociale en matière de prévention ou de couverture du risque.

\section{Prévention}

Les moyens d'action des Caisses régionales, chargées de la prévention, sont améliorées :

a) les taux de cotisation, pour l'assurance " accidents du travail », seront de plus en plus calculés sur le coût réel des accidents de l'entreprise au lieu de l'être sur le coût moyen de la profession;

b) les possibilités d'accorder des ristournes ou d'imposer des cotisations supplémentaires sont élargies;

c) des mesures de prévention pourront être imposées à un employeur ou à un ensemble d'employeurs de la région;

d) la coordination de l'action de l'Inspection du Travail et de la Sécurité Sociale est renforcée.

\section{Couverture du risque}

Quelques aménagements tendent à mieux couvrir le risque « accident du travail " et allègent un peu la procédure. Citons la possibilité de demander des dommages, en cas de difficultés de reclassement ou de promotion, ou pour préjudice moral.

\section{RESPONSABILITÉS ET SANCTIONS}

Un long exposé, qui ne peut avoir place ici, serait nécessaire pour distinguer les responsabilités mises en cause par un accident du travail et éclairer la très vive polémique qui s'est ouverte au Parlement et se prolongera encore longtemps.

Pour bien voir la portée des textes, il faut rapprocher les articles 8,19 à 22 et 29 de la loi de 1976. Deux appréciations contraires sont exprimées. Les unes 
soulignent que les lois pénales existantes ne sont absolument pas modifiées et que la loi de 1976 ne fait qu'ajouter de nouvelles possibilités de sanctions. D'autres pensent que cette loi donne des apaisements aux représentants du patronat et que les nouvelles dispositions pénales pourraient bien être utilisées surtout à l'encontre des cadres et préposés.

Il faudra attendre quelques années pour savoir comment les tribunaux utiliseront l'arsenal qui leur est proposé par la loi de 1976. Je crains, pour ma part, que les cadres soient quelque peu malmenés. La jurisprudence bien établie jusqu'ici permettant de poursuivre un préposé ou un cadre, je ne pense pas qu'il était nécessaire " d'en rajouter ». Par contre, il me semble nécessaire de définir un statut légal des cadres de sécurité. Le Parlement l'a refusé. Ce statut des Ingénieurs et Cadres de Sécurité est rendu indispensable par les nouvelles règles pénales. La Communauté Européenne le recommande et la France est devancée déjà par trois pays européens.

Voici donc quelques indications sur ces règles relatives aux responsabilités et sanctions :

\section{Faute personnelle}

La responsabilité de principe de l'employeur est remise en cause, mais déjà les tribunaux avaient bousculé cette notion.

\section{Non cumul des pénalités du Code Pénal et du Code du Travail}

Le tribunal ne pourra infliger les pénalités du Code Pénal simultanément avec celles du Code du Travail. Ce cumul était admis par la Cour de Cassation. Il $\mathrm{y}$ a là un allègement peu discutable.

\section{Principe de la personnalisation des peines}

La loi de 1976 crée une exception à ce principe, fondamental dans le Droit pénal français. Lorsqu'un préposé sera condamné, le tribunal pourra mettre le paiement de l'amende à la charge de l'employeur. Elle sera donc mise aux frais généraux de l'entreprise. La charge du préposé pourrait paraître adoucie, mais la loi est muette sur ce qu'il adviendra des pénalités éventuelles de prison ou de l'inscription au casier judiciaire.

\section{Pénalisation de l'entreprise}

En cas d'accident du travail, si le Tribunal ne retient pas la culpabilité d'une personne physique, il pourra condamner l'entreprise à exécuter un plan de travaux qui peut être très onéreux. Il peut, en effet, porter sur 5 ans et coûter au maximum cinq fois le montant de la cotisation annuelle d'assurance « accidents du travail ».

Pénaliser l'entreprise n'est pas une idée mauvaise en soi. Mais pourquoi mettre le juge devant l'alternative de condamner soit une personne - l'employeur ou, ne l'oublions pas, un cadre - soit l'entreprise. Ne faudra-t-il pas quelque courage au juge pour imposer un plan très coûteux ? 


\section{Interdiction d'exercer}

L'auteur d'une infraction pourra se voir interdire l'exercice de certaines fonctions pendant 5 ans.

\section{Faute inexcusable}

Lorsqu'une faute est grave et accomplie dans certaines circonstances bien défınies par la Cour de Cassation, elle est dite "inexcusable ". Elle peut entraîner une majoration de la rente au profit de la victime ou des ayants droit. Cette rente est payée par la Sécurité Sociale et récupérée sous forme de cotisation supplémentaire demandée à l'employeur.

Selon l'interprétation de la loi par la Cour de Cassation, l'interdiction de s'assurer contre ce risque financier était jusqu'ici absolue. La loi de 1976 interdit de s'assurer contre les conséquences de sa propre faute inexcusable. Elle ouvre à l'employeur la possibilité de s'assurer contre les charges de la faute inexcusable de ses préposés. Il s'ensuit que les victimes auront de meilleures chances de dédommagement, mais aussi que les préposés seront sans doute plus exposés puisque assurés et solvables.

\section{CONCLUSION}

Nous avons vu que pour les règles sur les responsabilités et les pénalités, la plupart immédiatement applicables, il nous faudra quelques années avant de savoir quel usage en auront fait les magistrats. Toutes les autres règles peuvent être grandement modelées ou infléchies par les décrets d'application. Aussi, l'impression qui domine ce tour d'horizon est qu'il faut attendre quelque peu pour connaître la portée réelle de la nouvelle loi. 